\title{
Deformation and failure of polymer bonded explosives under diametric compression test
}

\author{
Pengwan Chen ${ }^{\mathrm{a}, *}$, Huimin Xie ${ }^{\mathrm{b}}$, Fenglei Huang ${ }^{\mathrm{a}}$, Tao Huang ${ }^{\mathrm{a}}$, Yansheng Ding ${ }^{\mathrm{c}}$ \\ ${ }^{a}$ National Key Laboratory of Explosion Science and Technology, Beijing Institute of Technology, Beijing 100081, China \\ ${ }^{\mathrm{b}}$ Department of Engineering Mechanics, Tsinghua University, Beijing 100084, China \\ ${ }^{\mathrm{c}}$ Institute of Mechanics, Chinese Academy of Sciences, Beijing 100080, China
}

Received 9 November 2005; accepted 23 December 2005

\begin{abstract}
The tensile deformation and failure of polymer bonded explosives (PBXs), a particulate composite, is studied in this paper. Two HMX-based PBXs with different binder were selected for study. A diametric compression test, in which a disc-shaped specimen is loaded diametrically, was chosen to generate tensile failure in the materials. The quasi-static tensile properties and the tensile creep properties were studied by using conventional displacement transducers to measure the lateral strain along the horizontal diameter. The whole-field in-plane creep deformation was measured by using the technique of high resolution moiré interferometry. Real time microscopic examination was conducted to monitor the process of deformation and failure of PBXs by using a scanning electron microscope equipped with a loading stage. A manifold method (MM) was used to simulate the deformation and failure of PBX samples under the diametric compression test, including the crack initiation, crack propagation and final cleavage fracture. The mechanisms of deformation and failure of PBXs under diametric compression were analyzed. The diametric compression test and the techniques developed in this research have proven to be applicable to the study of tensile properties of PBXs.
\end{abstract}

(C) 2006 Elsevier Ltd. All rights reserved.

Keywords: Polymer bonded explosives; Diametric compression test; Deformation and failure; Microstructure; Moiré interferometry

\section{Introduction}

Polymer bonded explosives are highly particle filled composite materials comprised of $90-95 \%$ by weight of powerful secondary explosive crystals held together by a polymeric binder (5-10\% by weight). They are used in a wide variety of applications, ranging from rocket propellants to the main explosive charge in conventional munitions. Of great concern to explosive formulations is the possibility of accidental explosion

\footnotetext{
* Corresponding author. Tel.: +86 10 68912858; fax: +86 10 68461701.

E-mail address: pwchen@bit.edu.cn (P. Chen).
}

during manufacture, transport or handling. The mechanical properties of explosive materials subjected to a range of conditions are important criteria to determine a safe working life. Failure in particulate composites is heavily dependent upon the interaction between the particles and the matrix, specifically on the separation of particles and binder. In addition, failure also depends on the volume ratio of particle to matrix. With a much higher concentration of particles than conventional structural polymers (usually in the range of $15-40 \%$ ), PBXs may exhibit different failure mechanisms to conventional particulate composites. The study of mechanical properties and the failure mechanisms of explosives have drawn tremendous attention in recent years [1-4]. 
In view of the hazardous nature of the explosive materials and the lack of facilities for handling large quantities, tests sometimes have to be performed on small samples. Low strengths and safety concerns bring additional difficulties in conducting such tests for explosive materials, sometimes even making conventional tests, e.g. uniaxial tests impossible. One of the most suitable tests for this is a diametric compression test, known as the Brazilian test or the indirect tensile test. Diametric compression tests, in which a disc of material is loaded diametrically in compression, can be used to generate tensile stress and estimate the tensile failure stress [5] and study the creep deformation [6] of a material without the inconvenience of uniaxial dumbbell testing. Application of this method to explosives has been undertaken by Johnson [7,8], in which the tensile strain was measured by displacement transducers. More recently, some optical techniques, including laser speckle photography and moiré interferometry have been used to measure the whole-field deformation in diametric compression tests and study the quasi-static tensile properties of PBXs [9-11]. At present, application of these optical techniques to the study of the creep deformation of explosives is rarely found in the literature.

In the present work, the deformation and failure of polymer bonded explosives under a diametric compression test was experimentally studied by using different techniques including high resolution moiré interferometry and real time microscopic examination. In addition, the failure process of PBXs was numerically studied by using the manifold method. The results presented in this paper may provide some further insights into the deformation and failure mechanisms of explosives.

\section{Experimental}

\subsection{Materials}

Two HMX-based PBXs designated as PBX-A and PBX-B were investigated in the experiments. Both the PBXs contained HMX 94.5-95.0\% and a polymeric binder $5.0-5.5 \%$ by weight, however different binders were used. In addition, a PBX simulant, which consists of $89 \% \mathrm{KCl}$ and $11 \%$ polymeric binder by weight, was used for the creep study by moiré interferometry. The PBXs and the simulant were pressed in a steel die under different temperatures and pressures to produce disc shaped samples. For hot pressing, the temperature was $100{ }^{\circ} \mathrm{C}$ and the duration of pressing was $1.5 \mathrm{~h}$. For cold pressing, the temperature was $20^{\circ} \mathrm{C}$. Different pressing pressures were used, ranging from 50 to $200 \mathrm{MPa}$. The PBX simulant was only used in moiré interferometry tests. The disc sample size for real time microscopic examination and moiré interferometry was $\phi 20 \mathrm{~mm} \times$ $6 \mathrm{~mm}$, and the size for other experiments was $\phi 20 \mathrm{~mm} \times 10 \mathrm{~mm}$.

\subsection{Diametric compression test and microscopic examination}

Fig. 1 shows the loading geometry of the diametric compression test. The imposition of a compressive load, $P$, from the two anvils on a disc of material causes a tensile failure on the vertical loading axis. According to simple elasticity theory, the stress distribution along the loading diameter is described by the following formulae

$\sigma_{x}=\frac{2 P}{\pi D t}$

$\sigma_{y}=-\frac{2 P}{\pi t}\left(\frac{2}{D-2 y}+\frac{2}{D+2 y}-\frac{1}{D}\right)$

$\tau_{x y}=0$

where $D$ and $t$ are the sample diameter and the thickness, respectively. The lateral displacements across the horizontal diameter were measured by two extensometers. Both the quasi-static tensile deformation under different strain rates and the creep deformation under different stress levels were measured.

In general, failure occurs along the loading axis in a diametric compression test, which brings convenience for real time microscopic examination of the failure process. In order to do this, a diametric compression test was also carried out in a SEM equipped with a loading stage. In real time microscopic examination,

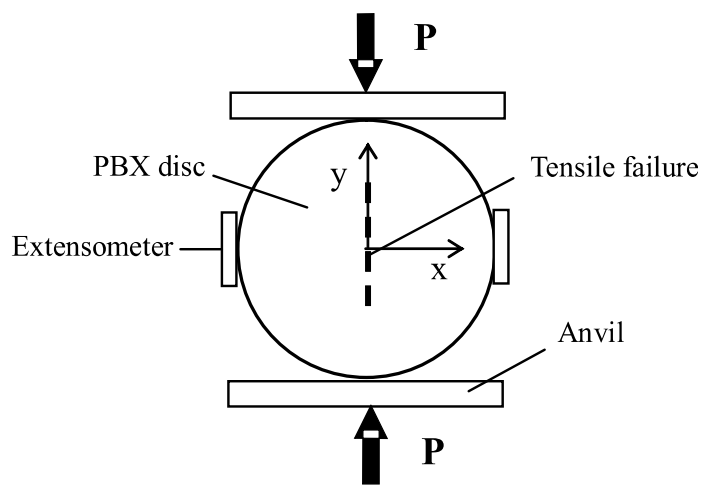

Fig. 1. Diametric compression test geometry. 


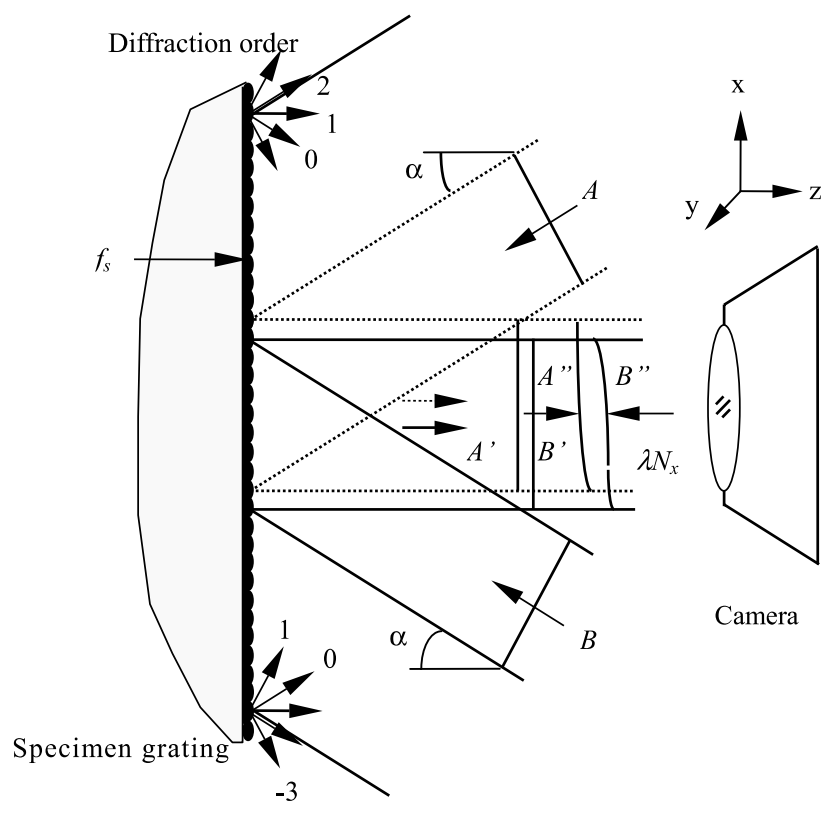

Fig. 2. The beam path of the moiré interferometry.

the images of the central region of the sample disc were recorded before loading, and then load was applied slowly. When the first sign of failure appeared, loading was stopped and the loading anvils held at constant separation as the cracks grew and the load relaxed. In this way, the failure could be observed and followed.

Low toughness and safety concerns bring additional difficulties in preparing the PBX samples for microscopic examination. The method of sample preparation here is similar to that of Rae et al. [12]. Samples were first ground using standard fine silicon carbide papers (800 grit) to obtain a flat surface. Final polishing was carried out in an automatic polishing machine using $1 \mu \mathrm{m}$ alpha alumina powder while being lubricated with distilled water. To avoid bringing additional unexpected damage to the samples during polishing, especially the fractured samples, the samples were first potted in commercial low-viscosity epoxide mounts with traditional amine hardening agent and then cured.

\subsection{Creep deformation measurement by moiré interferometry}

High resolution moiré interferometry is a sensitive coherent optical technique, which allows the measurement of in-plane displacements with sub-wavelength sensitivity $[13,14]$. In this method, a high frequency crossed-line diffraction grating is replicated on the surface of the specimen, and it deforms together with the underlying specimen. The two incident beams are symmetrical with respect to the normal to the specimen grating as shown in Fig. 2. When the incidence angle $\alpha$ of the collimated beams satisfies $\alpha=\arcsin \left(\lambda f_{\mathrm{s}}\right)$, where $\lambda$ is the wavelength of light and $f_{\mathrm{s}}$ is the frequency of the specimen grating, the +1 and -1 diffraction orders of initial beams $A$ and $B$ emerge along the normal to the specimen surface, respectively. Before the deformation of the specimen, the grating is perfect and the two diffracted beams have plane wavefronts $A^{\prime}$ and $B^{\prime}$. When the specimen is loaded, the wavefronts become warped $A^{\prime \prime}$ and $B^{\prime \prime}$ because of the deformation of the specimen grating. The two wavefronts interfere with each other in the image plane and the CCD records the moiré fringe patterns corresponding to $u$ and $v$ displacements.

The displacement components $u$ and $v$ are obtained from the fringe order $N_{x}$ and $N_{y}$ by

$u=\frac{N_{x}}{2 f_{\mathrm{s}}}$

$v=\frac{N_{y}}{2 f_{\mathrm{s}}}$

and the in-plane strains by

$\varepsilon_{x}=\frac{1}{2 f_{\mathrm{s}}} \frac{\partial N_{x}}{\partial x}$

$\varepsilon_{y}=\frac{1}{2 f_{\mathrm{s}}} \frac{\partial N_{y}}{\partial y}$ 


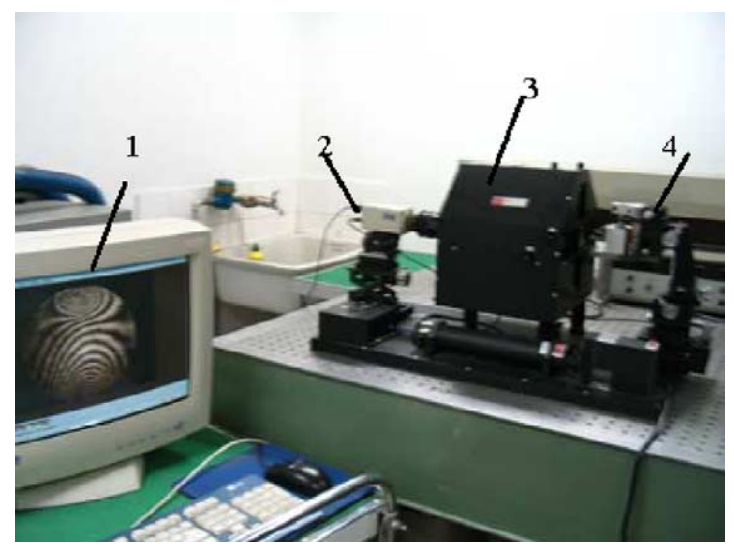

Fig. 3. Photography of the moiré interferometry system. 1, Computer; 2, $\mathrm{CCD} ; 3$, moiré interferometer; 4 , the loading stage.

Fig. 3 shows the photograph of experimental equipment, including the loading system, moiré interferometer, $\mathrm{CCD}$ and the computer. A loading stage was developed, which allows adjustment in six axes to align the specimen grating. The moiré interferometer is equipped with a $\mathrm{He}-\mathrm{Ne}$ laser, and the inside light path is arranged as in Fig. 2, providing the reference grating along horizontal and vertical directions to produce $u$ and $v$ displacement fringe patterns. The specimen grating is a diffraction grating with line density of 1200 lines/mm.

\section{Failure simulation by manifold method}

\subsection{Brief introduction to manifold method}

The manifold method proposed by Shi [15] is a new numerical method, which provides a unified framework for solving problems with both continuous and discontinuous media. By employing the concept of cover and two sets of meshes, the manifold method combines the advantages of FEM and discontinuous deformation analysis (DDA). It can not only deal with discontinuities, contact, large deformation and block movement as DDA, but also provide the stress distribution inside each block as accurately as FEM can. The numerical model of the original MM possesses only first-order accuracy, leading to dissatisfaction in simulating problems that need high accuracy in displacement and stress distribution. To overcome this and expand the applicability of MM, Zhang et al. [16] developed the second order manifold method with six node triangle mesh. In the present paper, the second order MM was applied to study the failure process of PBXs under quasi-static diametric compression test.
For basic theories of $\mathrm{MM}$, please refer to the Refs. $[15,16]$.

\subsection{Criterion of crack initiation and propagation}

PBXs exhibit similar mechanical properties to rock, with small failure strains and compressive strengths much higher than tensile strengths. In view of these similarities, a criterion of crack initiation and propagation proposed by Zhang et al. [17] originally for rock is used in this paper. The criterion is briefly described as follows.

Different criteria are used in the initiation of new cracks and the growth of existing cracks. For the initiation of new cracks, a stress-based criterion, MohrCoulomb's Law with three parameters, is considered. It is assumed that new cracks initiate if: (a) the first principle stress is larger than the tensile strength of the material, or (b) the maximum shear stress is larger than the shear strength of the material. The failure criterion can then be expressed as

Tensile failure

$\sigma_{1}=T_{0}$

Shearing failure

$$
\begin{aligned}
\frac{\sigma_{1}-\sigma_{3}}{2}= & C, \text { if } \frac{\sigma_{1}+\sigma_{3}}{2}>0 \text { and } 0<\sigma_{1}<T_{0} \\
\frac{\sigma_{1}-\sigma_{3}}{2}= & C \cos \phi-\frac{\sigma_{1}+\sigma_{3}}{2} \sin \phi, \text { if } \frac{\sigma_{1}+\sigma_{3}}{2} \\
& <0 \text { and } 0<\sigma_{1}<T_{0}
\end{aligned}
$$

where $\sigma_{1}$ and $\sigma_{3}$ are the first and third principal stresses, $T_{0}$ is the tensile strength, $C$ is the cohesion and $\phi$ is the friction angle.

For the existing cracks, the fracture toughness $K_{\text {Ic }}$ of the material is taken as the fracture criterion, and the maximum circumferential stress theory is adopted to determine the direction of crack growth measured from the current crack line $\theta$

$K_{\mid} \sin \theta+K_{||}(3 \cos \theta-1)=0$

The fracture criterion for a mixed mode problem takes the form:

$\cos \frac{\theta}{2}\left(K_{\mathrm{I}} \cos ^{2} \frac{\theta}{2}-\frac{3}{2} K_{\Pi} \sin \theta\right)=K_{\mathrm{IC}}$

The material parameters of PBXs used in the calculation model are: elastic modulus $E=2 \mathrm{GPa}$, Poisson's ratio $\nu=0.3$, unit mass $\rho=1.83 \times 10^{3} \mathrm{~kg} / \mathrm{m}^{3}$ tensile strength $T_{0}=2 \mathrm{MPa}$; fracture toughness 
$K_{\mathrm{IC}}=0.2 \mathrm{MNm}^{3 / 2}$; cohesion $c=5 \mathrm{MPa}$; friction angle $\phi=30^{\circ}$ The diameter of the specimen is $20 \mathrm{~mm}$, and the specimen is assumed to be in a plane stress condition.

\section{Results and discussion}

Fig. 4 shows the tensile stress strain curves of the two PBXs under a strain rate of $4.2 \times 10^{-4} \mathrm{~s}^{-1}$, in which curve $C$ denotes PBX-A pressed under $200 \mathrm{MPa}$ and $100{ }^{\circ} \mathrm{C}$, while curves $A, B, D$ and $E$ denote PBX-B pressed under different pressures (ranging from 50 to $200 \mathrm{MPa})$ and different temperatures $\left(100\right.$ and $\left.20^{\circ} \mathrm{C}\right)$. The stresses were calculated using formula (1). The results show that the tensile properties are greatly influenced by pressing pressure. The tensile strengths of PBX-B increase from 1.5 to 2.2 MPa with an increase of the pressing pressure from 50 to $200 \mathrm{MPa}$. Pressing temperatures have a greater influence on the tensile properties of PBXs than pressures. The tensile strength of cold pressed PBX-B $\left(20^{\circ} \mathrm{C}\right)$ is about $1.0 \mathrm{MPa}$, while the strength of $\mathrm{PBX}-\mathrm{B}$ pressed at an elevated temperature $100{ }^{\circ} \mathrm{C}$ is increased to $2.2 \mathrm{MPa}$. PBX-B exhibits a larger tensile strength and failure strain than PBX-A, implying that the mechanical properties of PBXs are largely influenced by the binder despite its low concentration. The tensile properties of PBXs are also influenced by strain rate. At a strain rate of $3.3 \times 10^{-5} \mathrm{~s}^{-1}$, the tensile failure stress of PBX-A is $0.90 \mathrm{MPa}$, compared with $1.4 \mathrm{MPa}$ at a strain rate of $4.2 \times 10^{-4}$.

Fig. 5 shows tensile creep curves of PBX-A obtained under stresses of 0.71 and $0.85 \mathrm{MPa}$ at the disc center, exhibiting classical creep behavior with three stages. The creep strain rate decreases gradually in the primary

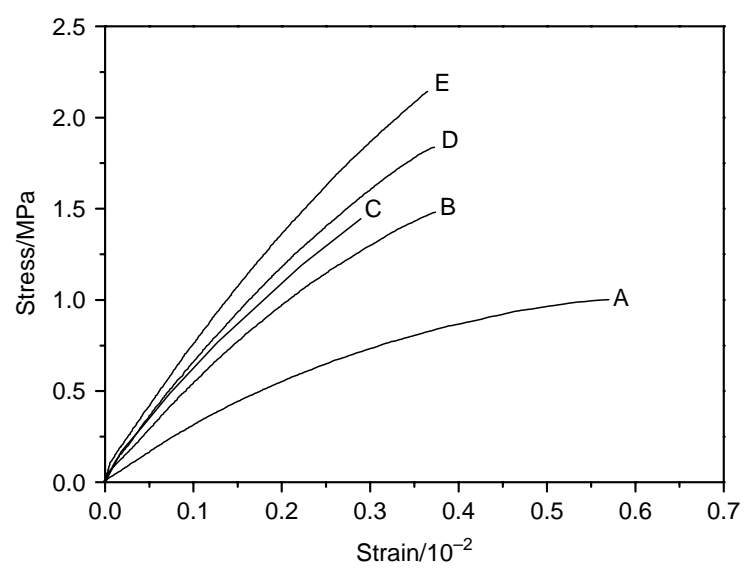

Fig. 4. Tensile stress strain curves of PBXs. (A) PBX-B (200 MPa, $\left.20{ }^{\circ} \mathrm{C}\right)$; (B) PBX-B $\left(50 \mathrm{MPa}, 100{ }^{\circ} \mathrm{C}\right)$; (C) PBX-A (200 MPa, $\left.100{ }^{\circ} \mathrm{C}\right)$; (D) PBX-B (100 MPa, $\left.100{ }^{\circ} \mathrm{C}\right)$; (E) PBX-B (200 MPa, $\left.100{ }^{\circ} \mathrm{C}\right)$.

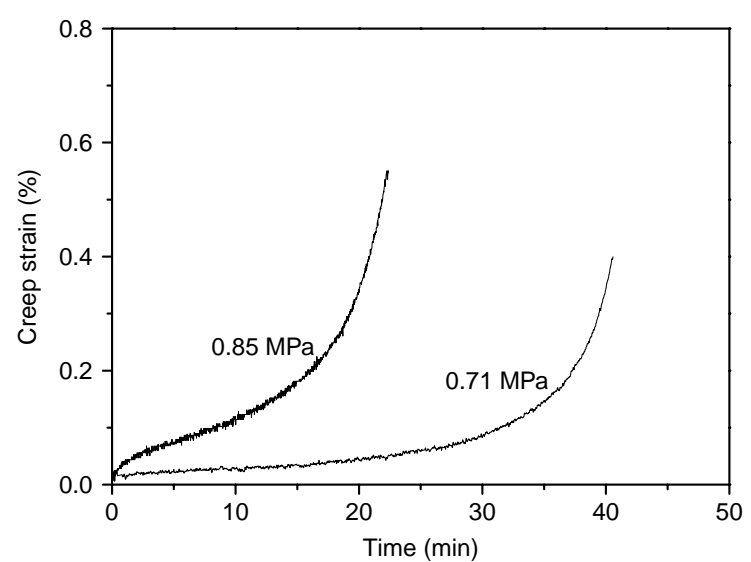

Fig. 5. Tensile creep curves of PBX-A.

stage, then remains nearly constant in the secondary stage (namely steady state creep stage), finally evolves into a rapid increase in the third stage (namely accelerating creep stage) until failure. The results also show that, with the increase of stress, the creep strain rate increases and the time period of the steady state creep stage decreases. The increase of stress also causes a decrease of the failure time and an increase of the failure strain. According to damage mechanics, the third stage is the result of interaction between the steady-state creep and the damage creep, and it is mainly the damage creep that contributes to the ultimate failure. The time dependent creep behavior of PBX is mainly due to the contribution of the binder, demonstrating that PBXs still exhibit viscoelastic properties despite a low concentration of binder.

The failure strains in quasi-static tension and tensile creep are relatively small, only about several millistrains, revealing the brittle nature of PBXs. The extremely high concentration of brittle explosive particles will account for this.

Real time microscopic examination reveals that different forms of failure including interfacial debonding, binder rupture and crystal fracture can be observed. The failure generally starts at several independent sites near the disc center, usually around the boundaries of the larger HMX filler particles, and forms microcracks. These microcracks link up into larger cracks and finally induce cleavage rupture of the samples. The initial damage, such as uncoating and debonding generated during the preparation of molding powder and pressing, are usually the origins of failure. Typically, the fracture route follows the boundaries of larger filler particles, while the crystal fractures are very few. However, crystal fractures do occur on some large particles when the particles are pre-damaged and their orientation 


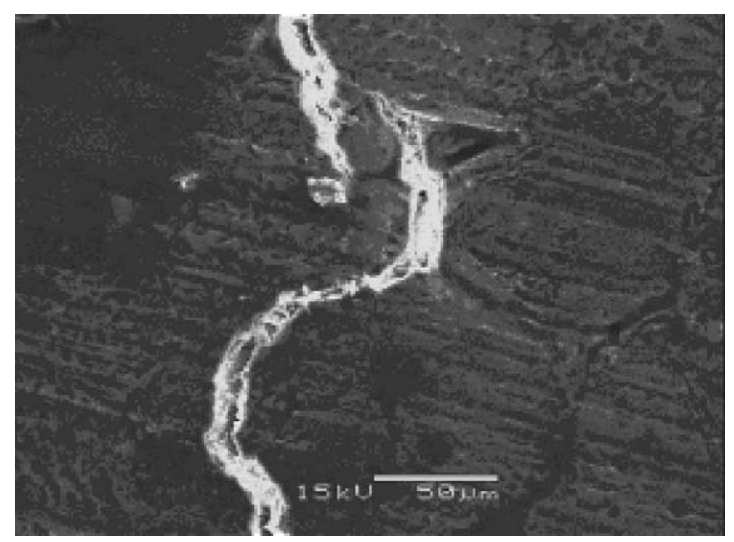

Fig. 6. Cracking along particle boundaries.

impedes crack growth. Figs. 6-8 show some results of real time microscopic examination of PBX-A. Fig. 6 shows that a crack is extending along the boundaries of particles. Fig. 7 shows some extended binder filaments bridging the crack surfaces, demonstrating that the binder undergoes considerable deformation. Please note that some micron-sized particles of filler can be observed within the network of binder filaments. Fig. 8 shows an image of cracking along large particles with the presence of numerous binder filaments spanning the crack. The upper large particle (labelled as A) with the presence of initial microcracks is fractured into several pieces, while the lower large particle (labelled as B) without the initial microcracks retains its integrity. The microcracks in large particles are observed to be caused by pressing, which generates large contact stresses due to particle-to-particle contact and causes considerable crystal fractures $[18,19]$.

The fracture surfaces of PBX-A in creep and quasistatic tensile test are relatively rough, with some particles completely pulled out. Larger particles exhibit clean crystal faces due to the debonding of binder from particles. In contrast, the finer particles appear rough

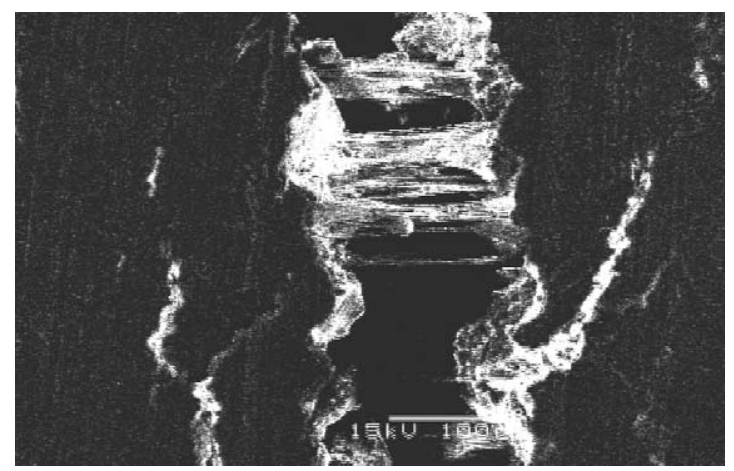

Fig. 7. Binder filaments bridging crack walls.

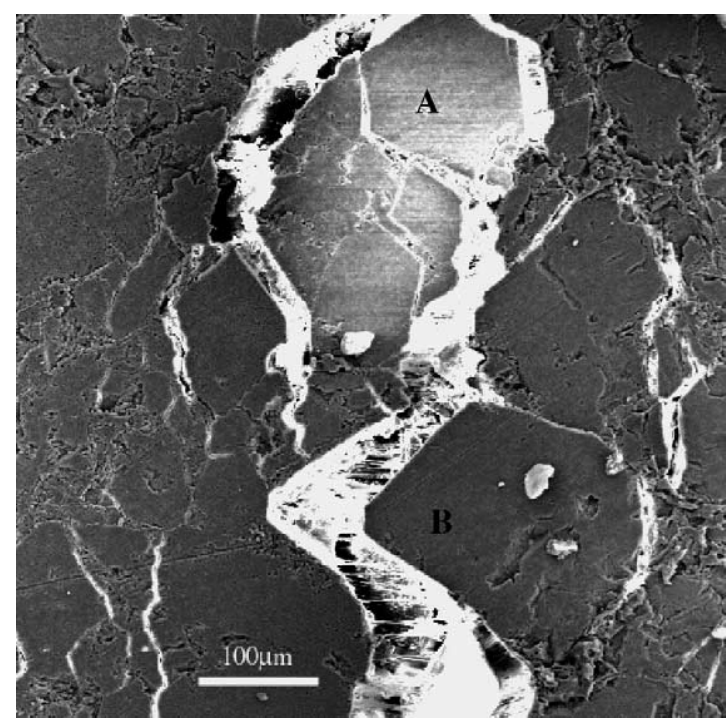

Fig. 8. Cracking along large particles with an evidence of crystal fractures.

having binder fibrils fractured due to extensive deformation. Fig. 9 shows a typical fractograph of PBX-A under quasi-static diametric compression test, in which both clean crystal surfaces and fractured binder can be observed. Examination of fracture surfaces also shows that the failure predominantly follows the boundaries of the explosive fillers due to interfacial debonding with few fractured crystals. Some microcracks can also be observed on some large particles (indicated by arrows) which considering the low stress amplitude applied in the test, is believed to be caused by pressing. Compared with PBX-A, the fracture surfaces of PBX-B are relatively smooth with more crystal fractures observed, exhibiting better

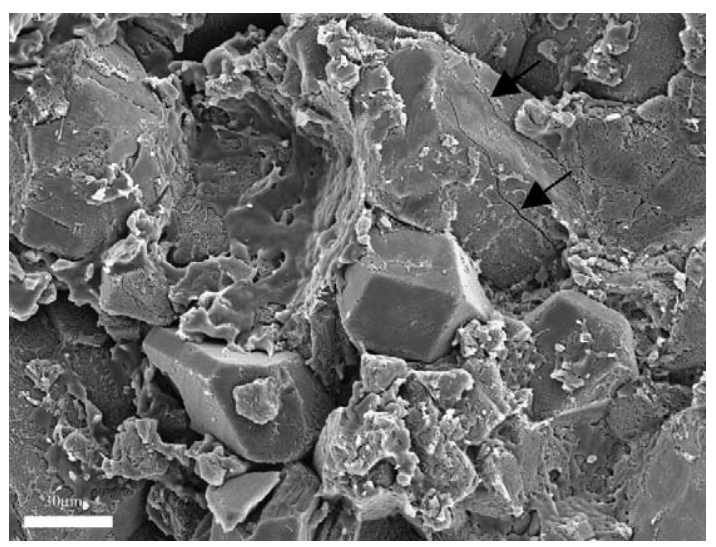

Fig. 9. A typical fractograph of PBX-A under quasi-static diametric compression test. 


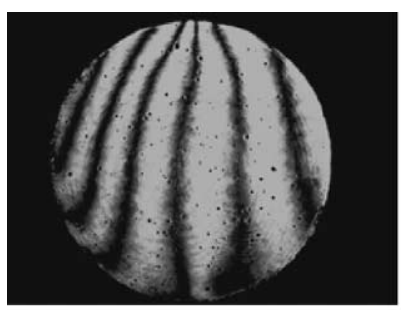

(a)

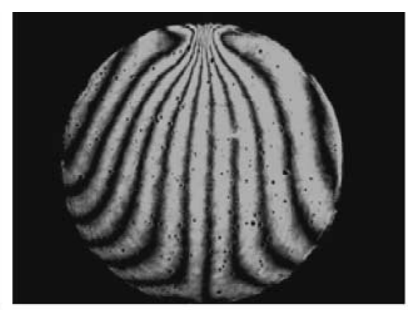

(b)

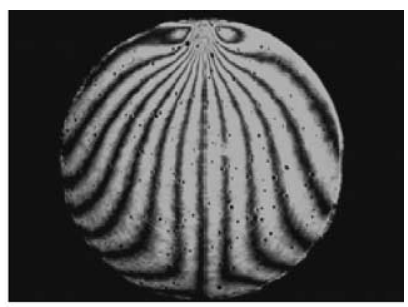

(c)

Fig. 10. $u$ field moiré patterns; (a) $t=0 \mathrm{~min}$; (b) $t=120 \mathrm{~min}$; (c) $t=825 \mathrm{~min}$.

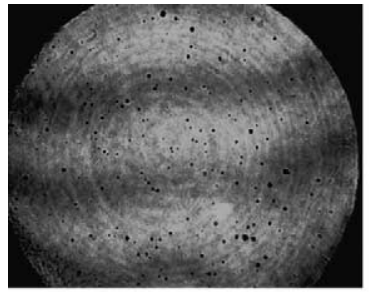

(a)

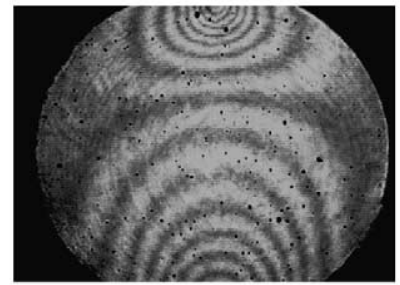

(b)

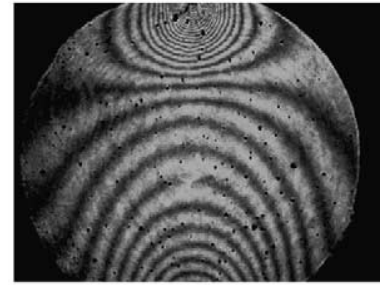

(c)

Fig. 11. $v$ field moiré patterns; (a) $t=0 \mathrm{~min}$; (b) $t=30 \mathrm{~min}$; (c) $t=696 \mathrm{~min}$.

interfacial bonding than PBX-A, which is consistent with the mechanical tests above.

Figs. 10-13 show the results of moiré interferometry tests for the PBX simulant. Figs. 10 and 11, respectively, show the displacement fringe patterns in the horizontal and vertical directions, namely $u$ and $v$ field moiré patterns, in which the applied stress $\sigma_{x}$ at the disc center is $0.98 \mathrm{MPa}$. The pressing pressure and temperature of the disc samples are $200 \mathrm{MPa}$ and $100^{\circ} \mathrm{C}$, respectively.

Fig. 12 shows the creep curves of two samples in the $x$ direction at the disc center derived from the moire patterns, in which curve 1 represents the sample pressed under $200 \mathrm{MPa}$ and $100{ }^{\circ} \mathrm{C}$ and curve 2 represents the sample pressed under $60 \mathrm{MPa}$ and $100{ }^{\circ} \mathrm{C}$. The applied stress $\sigma_{x}$ for the two samples is $0.98 \mathrm{MPa}$. In the figures, the asterisk represents the actual strains obtained in the experiment and the continuous curves represent the fitted curves (the creep curves in polynomial approximation of six orders). The creep strain of curve 2 is higher than that of curve 1 at the same time, demonstrating that a higher pressure generates better mechanical properties and enables the samples to exhibit better creep resistance. The results are consistent with the tensile test results above. Fig. 13 shows the creep curve of the sample pressed under $200 \mathrm{MPa}$ and $100{ }^{\circ} \mathrm{C}$ in the direction $y$ at the disc center, in which the corresponding stress calculated by formula (2) is $2.93 \mathrm{MPa}$.

Compared with the typical creep curve of a pure polymer and the lateral creep curves in Fig. 5, a repeating strain increase stage can be observed, in which the creep strain rate repeatedly changes from a decrease to an increase. This phenomenon can be observed more obviously in curve 1 of Figs. 12 and 13.

We speculate that the repeating increase stage is the results of interaction between creep and damage, which can be explained from the microstructure and the internal damage. Pressed PBXs are a highly particlefilled composite with random distribution of initial damage in the forms of interfacial debonding, crystal fractures, etc. as observed in microscopic examination. Some of the initial damage can be activated and some new damage can be generated at a relatively low stress

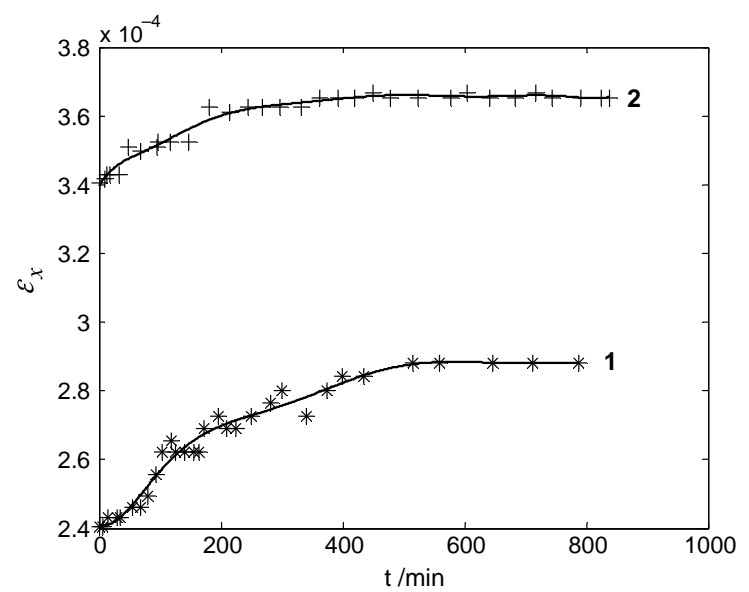

Fig. 12. Creep curves of the $\mathrm{PBX}$ simulant at the disc center in the direction $x$. Curve $1,200 \mathrm{MPa}, 100{ }^{\circ} \mathrm{C}$; curve $2,60 \mathrm{MPa}, 100{ }^{\circ} \mathrm{C}$. 


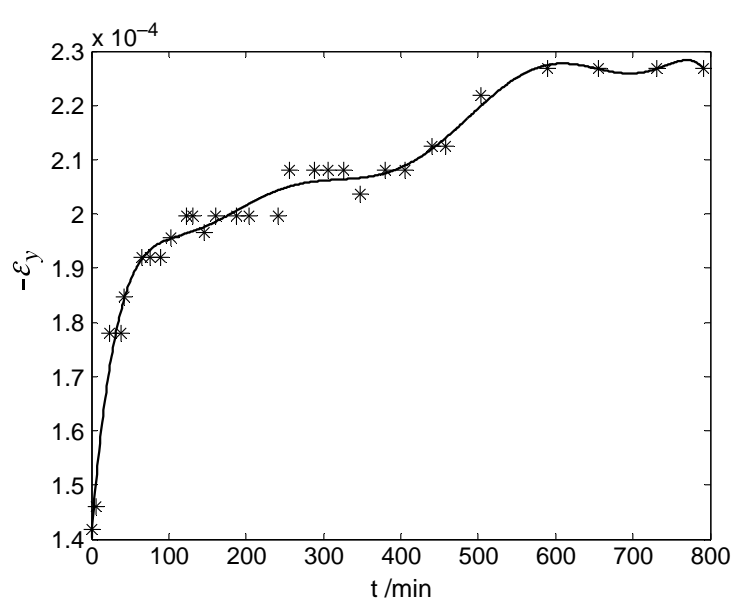

Fig. 13. A creep curve of the PBX simulant in the direction $y$.

and strain level according to the acoustic test results of PBXs [20], which can be further supported by the existence of a subcritical fracture in PBXs observed in our experiments [21]. In addition, a microcrack may propagate at different rates with respect to different failure forms. It is easier for a microcrack to propagate along the boundary of particles through interfacial debonding than through binder rupture, especially for particles with initial debonding and initial uncoating. Sometimes a microcrack may be impeded by a particle due to its orientation. Randomness of damage and various modes of propagation of damage (microcracks) are assumed to contribute to the occurrence of the repeating increase stage.

Fig. 14 shows the simulation results of the failure process of PBXs under a quasi-static diametric compression test by the manifold method, in which an image of a real fractured PBX sample [22] is shown for comparison (see Fig. 14(d)). The results show that the failure first initiates at the disc center and forms small cracks when the local tensile stress reaches the tensile

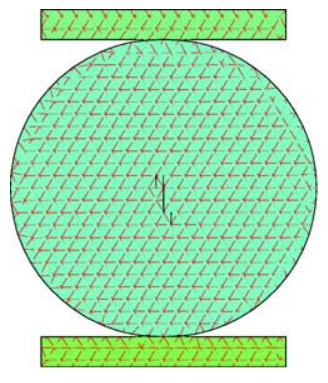

(a)

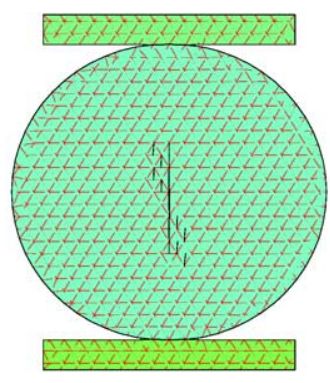

(b)

strength of PBXs. With the increase of applied load, the small cracks propagate along the vertical loading line towards the two ends, finally form a large vertical continuous crack and induce the cleavage rupture. It is noted that during the propagation of the main crack, some new small cracks form near the main crack due to the tensile stress exceeding the tensile strength, however, these cracks do not propagate noticeably. In general, the simulation is in good agreement with the theoretical analysis and experimental results. These results also demonstrate that the assumption of tensile failure of PBXs is rational.

\section{Conclusions}

Different failure forms including interfacial debonding, binder rupture and crystal fractures are observed under a diametric compression test. The initial failure tends to start at the disc center around the edges of larger filler particles and often occurs at several independent sites simultaneously. The fracture route tends to follow the boundary of filler particles with few fractured crystals.

Due to their extremely high concentration of particle filler, PBXs exhibit different tensile behavior from conventional particulate composites. In general, PBXs are brittle materials with low strengths and low fracture strains. The mechanical properties of PBXs are influenced by pressing pressures and pressing temperatures. In addition, their properties are largely influenced by the binder. Despite a low concentration of binder, the mechanical behavior of PBXs is rate dependent and time dependent. Due to the interaction between creep and damage, the creep curves of PBXs obtained by moiré interferometry exhibit a repeating strain increase stage. Applicability of the diametric compression test and the corresponding techniques developed in this

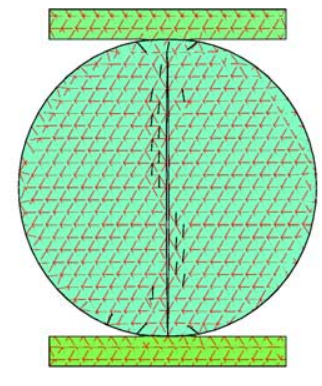

(c)

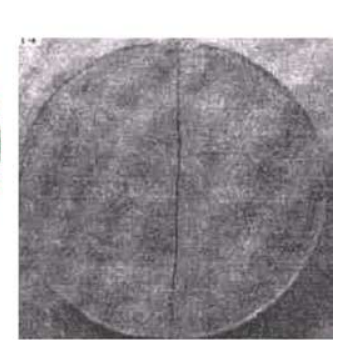

(d)

Fig. 14. Simulation results of the failure process of PBXs under diametric compression test, in which (d) shows the experimental results for comparison. 
paper to the study of the deformation and failure of PBXs is verified.

\section{Acknowledgements}

The authors of this paper acknowledge the financial support from the National Natural Science Foundation of China (contract number 10002022), Joint Foundation of Chinese National Natural Science Committee and Chinese Academy of Engineer Physics (contract number 10076021) and the Opening Funds from the National Key Laboratory of Explosion Science and Technology, Beijing Institute of Technology (contract number KFJJ03-1).

\section{References}

[1] D.A. Wiegand, Mechanical properties and mechanical failure of composite plastic bonded explosives and other energetic materials, in: Proceedings of the Eleventh International Detonation Symposium, Office of Naval Research, Portland, OR, 1998, pp. 744-750.

[2] G.T. Gray III, D.J. Idar, W.R. Blumenthal, C.M. Cady, P.D. Peterson, High- and low-strain rate compression properties of several energetic material composites as a function of strain rate and temperature, in: Proceedings of the Eleventh International Detonation Symposium, Office of Naval Research, Portland, OR, 1998, pp. 76-84.

[3] T. Kauly, T. Kimmel, Failure mechanism in PBX, in: Proceedings of the Energetic Materials, 29th International Annual Conference of ICT, Karlsruhe, Germany, 1998, p. 19.

[4] P.D. Peterson, K.S. Mortensen, D.J. Idar, B.W. Asay, D.J. Funk, Strain field formation in plastic bonded explosives under compressional punch loading, Journal of Materials Science 36 (2001) 1395-1400.

[5] H. Awaji, S. Sato, Diametral compressive testing method, Journal of Engineering Materials and Technology 101 (1979) 139.

[6] K.S. Chan, N.S. Brodsky, A.F. Fossum, S.R. Bodner, Creepinduced cleavage fracture in WIPP salt under indirect tension, Journal of Engineering Materials and Technology 119 (1997) 393-400.

[7] H.D. Johnson, Mechanical Properties of LX-10-1 Evaluated with the Diametric Disc Test, MHSMP-78-08, 1978.

[8] H.D. Johnson, Diametric Disc and Standard Tensile Test Correlation Study, MHSMP-81-22, 1981.

[9] S.J.P. Palmer, J.E. Field, J.M. Huntley, Deformation, strengths and strains to failure of polymer bonded explosives, Proceedings of the Royal Society of London Series A 440 (1993) 399.

[10] P.J. Rae, H.T. Goldrein, S.J.P. Palmer, J.E. Field, A.L. Lewis, Studies of the failure mechanisms of polymer-bonded explosives by high resolution moiré interferometry and environmental scanning electron microscopy, in: Proceedings of the Eleventh International Detonation Symposium, Snowmass, 1998, p. 66.

[11] H.T. Goldrein, J.M. Huntley, S.J.P. Palmer, M.B. Whitworth, J.E. Field, Optical techniques for strength studies of polymer bonded explosives, in: J.M. Short, D.G. Tasker, (Eds.), Proceedings of the Tenth International Detonation Symposium, Boston, MA, 1993, pp. 525-535.

[12] P.J. Rae, H.T. Goldrein, S.J.P. Palmer, J.E. Field, A.L. Lewis, Quasi-static studies of the deformation and failure of $\beta$-HMX based polymer bonded explosives, Proceedings of the Royal Society of London Series A 458 (2002) 743.

[13] D. Post, in: A.S. Kobayashi (Ed.), Moiré Interferometry, Handbook on Experimental Mechanics, Prentice-Hall, Eaglewood Cliffs, NJ, 1987.

[14] Huimin Xie, Satoshi Kishimoto, Jindong Li, Daqing Zou, Fulong Dai, Norio Shinya, Deformation analysis of laser processed grain oriented silicon steel sheet using moire interferometry, Materials Science Research International 5 (4) (1999) 291-295.

[15] G.H. Shi, Manifold method of material analysis, Proceedings of the Ninth Army Conference on Applied Mathematics and Computing, Minneapolis, MN, USA, 1991, pp. 51-76.

[16] G.X. Zhang, Y. Sugiura, H. Hasegawa, G. Wang, The second order manifold method with six node triangle mesh, Journal of Structural Mechanics and Earthquake Engineering, JSCE 696 (I58) (2002) 1-9.

[17] G.X. Zhang, Y. Sugiura, H. Hasegawa, F.Q. Chen, Crack propagation by manifold and singular boundary element method, Fifth International Conference on Computational Structures Technology and the Second International Conference on Engineering Computational Technology, 2000, pp. 61-68.

[18] C.B. Skidmore, D.S. Phillips, P.M. Howe, J.T. Mang, J.A. Romero, The evolution of microstructural changes in pressed HMX explosives, Proceedings of the Eleventh International Detonation Symposium, Office of Naval Research, Portland, OR, 1998, pp. 556-564.

[19] P. Chen, F. Huang, K. Dai, Y. Ding, Detection and characterization of long-pulse low-velocity impact damage in plastic bonded explosives, International Journal of Impact Engineering 31 (2005) 497-508.

[20] K. Ellis, C. Leppard, H. Radesk, Mechanical properties and damage evaluation of a UK PBX, Journal of Materials Science 40 (23) (2005) 6241-6248.

[21] D. Li, D. Xing, Y. Ding, X. Dong, P. Chen, W. Zhang, Y. Hao, S. Cheng, Experimental study on subcritical fracture of PBX material, Energetic Materials 12 (Suppl.) (2004) 687 (in Chinese).

[22] H. Song, Y. Hao, H. Dong, M. Wen, M. Li, A preliminary evaluation of tensile properties of small plastic bonded explosive sample by diametric disc test, Explosive and Shock Waves 21 (1) (2001) 35-40 (in Chinese). 